\title{
Ideas sobre la pedagogía de la seducción en el profesorado universitario
}

\author{
Amar Rodríguez, Víctor \\ Ideas sobre la pedagogía de la seducción en el profesorado universitario \\ Revista Educación, vol. 44, núm. 2, 2020 \\ Universidad de Costa Rica, Costa Rica \\ Disponible en: http://www.redalyc.org/articulo.oa?id=44062184025 \\ DOI: https://doi.org/10.15517/revedu.v44i2.39535
}

Esta obra está bajo una Licencia Creative Commons Atribución-NoComercial-SinDerivar 3.0 Internacional. 
Artículos científicos

\title{
Ideas sobre la pedagogía de la seducción en el profesorado universitario
}

\author{
Ideas about the Pedagogy of Seduction among University Teaching Staff
}

Victor Amar Rodríguez

Facultad de Educación. Departamento de Didáctica.

Universidad de Cádiz., España

victor.amar@uca.es

(iD http://orcid.org/0000-0001-9036-2651

\author{
DOI: https://doi.org/10.15517/revedu.v44i2.39535 \\ Redalyc: http://www.redalyc.org/articulo.oa?id=44062184025
}

Recepción: 05 Diciembre 2019

Aprobación: 09 Junio 2020

\section{Resumen:}

Es posible que haya escuchado hablar de pedagogía y seducción; no obstante, en la introducción se irá desglosando un parecer fundamentado sobre la pedagogía de la seducción. En este sentido, se propone un ensayo junto a una estrategia metodológica la cual sugiere la interpretación de algunas obras pictóricas para comprender un poco mejor la pedagogía de la seducción. A partir de resultados inspirados en tres acciones: la comunicación no verbal, la comunicación asertiva y la coreografía entre pantallas; habría que tener presente el saber interactuar con el cuerpo, del mismo modo que hace falta mantener una comunicación cercana con la empatía y en la serenidad, en la escucha activa y respetuosa; sin olvidar que el alumnado universitario se desenvuelve con las pantallas: las suyas y la que pueda utilizar el profesorado. Y, a modo de conclusión, toda esta propuesta de aproximación se establece sobre un diálogo simbólico en pro de mejorar la docencia universitaria.

Palabras clave: Pedagogía, Universidad, Seducción, Pantalla, Pedagogía de la seducción.

\section{Abstract:}

Perhaps you have heard of pedagogy and seduction; the introduction, nevertheless, presents a grounded breakdown of one opinion on the pedagogy of seduction. This essay presents a methodological strategy to better understand the pedagogy of seduction through interpretation of works of art. Results are based on three actions: non-verbal and assertive communication and choreography among screens. We must also remember to interact with our bodies, demonstrate empathy and serenity and be respectful and active listeners in order to engage students. College and university students tend to flourish through screens either their own or a screen that the professor or lecturer may use to complement class instruction. The ultimate goal is to nourish symbolic dialogue and, thereby, enhance the classroom experience for college and university level students.

KEYWORDS: Pedagogy, University, Seduction, Screens, Pedagogy of Seduction.

\section{INTRODUCCIÓN A LA NOMENCLATURA}

El profesorado universitario: ¿cuántas veces se ha detenido a pensar en indagar las posibles maneras de atraer la mirada o el interés de su alumnado? ¿En cuántos momentos ha pensado en los problemas de la actual Universidad? O bien, ¿qué podría hacer para mejorar la docencia? Tal vez, sea la forma menos adecuada de comenzar un artículo de esta naturaleza. Pero, no obstante, se considera que puede ser una de las más apropiadas. Sin embargo, esta es la manera que se ha encontrado para compartir las inquietudes sobre una posible realidad invasiva como puede ser la falta de interés o desmotivación por parte del alumnado que accede a la formación universitaria (Carrasco, 2015). Ya no se han de tratar como niños, pues no lo son. Tampoco se pueden dejar a su libre albedrío, ya que se podría incurrir en algún tipo de dejación de las funciones como docentes. El equilibrio se apodera de esta propuesta a modo de ensayo con la pretensión de promover ideas para el debate sobre una posible pedagogía de la seducción en el profesorado universitario (Ruiz, 2006; Perrenoud, 2001). Y contará como estrategia metodológica el recurso de analizar las obras pictóricas para explicar la pedagogía de la seducción. 
En cierto modo, se está pensando en un profesorado vinculado, principalmente, con las ciencias sociales y con las tecnologías (Ertmer y Ottenbreit, 2010). Sin embargo, habrá aspectos compartidos en este trabajo que pueden hacerse extensibles a las otras áreas de conocimiento no necesariamente vinculadas con lo social. Con todo, no estaría de más en esta introducción empezar a hablar sobre qué se entiende sobre pedagogía y por seducción. Quizás, sea importante aclarar los términos para comenzar a compartir los mismos significados y poder, entonces, iniciar el propio desarrollo del artículo.

Es decir, pedagogía es el arte de enseñar (Meirieu, 2018). Ahora bien, se recuerda que en la contemporaneidad existen herramientas persuasivas que facilitan la labor de enseñar; lo cual no es una garantía de éxito. Y, con el propósito de ir centrando la temática, se ha reducido bastante la definición de pedagogía a la de arte. Por ello, se interpreta como arte a aquella acepción que lo relaciona con las maneras de.... Se explica un poco mejor. Por ejemplo, los marineros tienen artes para pescar según el pez van a capturar. Saben cuál es el arte que han de utilizar dependiendo de la temperatura del mar o el viento predominante, la profundidad del caladero... Es decir, el oficio les dicta aquella determinada manera de pescar. No van al azar ni mucho menos es algo aleatorio.

Entonces, para la pedagogía, habría que tener presente el hecho de conocer las maneras de... . No es igual impartir clases en primero que en cuarto de carrera. Pero tampoco es lo mismo dar aulas en una troncal que en una optativa, sea con una intención teórica o práctica, en gran grupo en pequeño grupo... Para solucionar estas situaciones que, a priori, pueden resultar anecdóticas... La pedagogía es el arte de exponer con claridad y atendiendo a la diversidad, con la finalidad de enseñar. Un ejercicio compartido donde intervienen no solo el alumnado y el profesorado sino un sinnúmero de elementos, junto al contexto, que interfieren o ayudan en el ejercicio de la seducción. Díaz (2019) considera sobre el discurso pedagógico que se trata de: "un medio muy importante en la configuración del orden, relación e identidad dentro de y entre individuos y grupos sociales" (p. 21).

Ahora bien, el siguiente término que se debería aclarar sería el de la seducción. Probablemente, el primer pensamiento sobre seducción, la mayoría de las veces, se asocia a situaciones íntimas o privadas. Pero es un error, pues la seducción se produce, explícitamente, en cualquier momento en que se procure una cierta aproximación intencionada a alguien o algo. Se puede seducir con la imagen, con el discurso, con la manera de expresarse o comportarse. El proceso de aproximación se inicia, tal vez, de manera espontánea, pero a partir de este momento, se ha de evidenciar los conocimientos en cuanto a la pedagogía de la seducción. Es decir, ante un auditorio compuesto por el alumnado, expectante por escuchar, se hace la siguiente pregunta: ¿cuántos aspectos existen en el aula para que el estudiantado se disperse? Cabría considerar desde el horario intempestivo de comienzo de las clases a las horas acumuladas en la facultad. A la postre, el éxito como docente, se evidencia cuando se oiga decir:

He sido seducido, pero ello me ha permitido comprender esto o aprender aquello y lo que sé lo puedo identificar, reutilizarlo fuera del contexto de su aprendizaje; ahora soy yo el dueño y, aunque lleve aún durante un cierto tiempo la huella de la(s) persona(s) mediante la(s) cual(es) he llegado a obtener estos conocimientos, soy capaz de confrontarlos con nuevas situaciones (Meirieu, 1987, p. 90).

Una estrategia, en apariencia simple, sería la de seducir; no obstante, para otros se trata de un arte (Grene, 2008). O sea, cualquier actitud de aproximación comienza a romper el hielo que pueda existir entre estudiantes y profesorado. Hay quien recurre a la comicidad, al despiste o a la sobriedad para atraer la atención del alumnado. Son maneras de presentarse en público que, a su vez, es el modo que se ha encontrado para representar el papel de actor en el arte de enseñar. Pero no se puede olvidar que existen elementos intrínsecos y extrínsecos que restan poder a la representación pública. Entre ellos, se puede incluir los que ya pueda traer el propio aprendiz de su casa o los que ya se les haya incrementado en la facultad. Además, cabría añadir las pantallas que distorsionan el poder de seducción (Marcelo, Yot y Mayor, 2015). En cierto modo, se suscribe que las pantallas sí que restan protagonismo al docente, pero no capacidad de seducción con respecto al alumnado. Se explican un poco mejor. 
Las pantallas son recursos, pues pueden facilitar al alumnado, entre otras cosas, el hecho de consultar aspectos de los contenidos que les interesan o no les han quedado claros. Pero, también, se ha de recordar que las redes sociales forman parte de su cotidianeidad (Alonso y Alonso, 2014; Rodríguez, López y Martín, 2017). Probablemente, y de manera inevitable, poco o nada se podrá hacer sobre el poder persuasivo que tienen las pantallas. La hipersimplificación junto con la inmediatez de las tecnologías son dos aspectos que irrumpen en la dinámica de aula; pese a que ambas se conforman como "compañeras de viaje" (Ceprián, 2019, pp. 123-127). El alumnado está, supuestamente, conectado a las enseñanzas, pero de repente suena, en el mejor de los casos vibra, su teléfono móvil y, por ejemplo, ¿cuánto tiempo tardará en consultar el WhatsApp y responder al mensaje?

Antes de entrar en confrontación con las evidencias de comunicación en el siglo XXI, a través de los dispositivos inalámbricos, cabría recordar que el teléfono móvil es una herramienta inteligente (Brazuelo, 2015). Ya no solo es un recurso sino un útil que además de informar y comunicar, posee la vertiente de favorecer las relaciones (Lazos y Gabels, 2016). Y no cabe estar en continua lucha con el alumnado, advirtiéndoles con un apaguen sus teléfonos, por favor [o] a la siguiente persona que le vea con el teléfono en sus manos, lo expulso del aula. No debe olvidarse que se está en la Universidad con personas que desean formarse (Krücken, 2014).

Antes de sostener la confrontación, no sería más ventajoso apropiarse de aquel pensamiento que dice: Si no puedes con tu enemigo, únete a él. Claro que, en ocasiones, cuesta admitir que el y la estudiante está atendiendo a su teléfono móvil en clase e, incluso, se interpreta como una falta de respeto. Pero, igualmente, el alumnado no viene solo al aula de la facultad con sus apuntes o libros. Ahora le acompaña un útil que deposita sobre su mesa que, a veces, está silenciado; lo que no significa que esté en silencio pues, el alumnado, tiene retorno de su presencia y actividad. Sin embargo, otros más listos para evitar la mirada molesta del profesorado, ocultan la presencia de su Smartphone (Brazuelo, Gallego y Cacheiro, 2017); pero todos se sabe que está ahí, languideciendo, pues no está apagado sino en modo vibración.

Ante tan atractiva, personal y versátil herramienta del siglo XXI, ¿qué se puede hacer? No se diga que nada. Siempre se puede hacer algo. Cabría establecer un gratificante consejo: Unirse a las herramientas de la contemporaneidad. Hacer la más elemental teoría de la pedagogía de la seducción. $\mathrm{O}$ sea, el arte de enseñar aproximándose. Dejar de lado la lucha contra las pantallas. Ser seductores y, también, dejarse seducir.

\section{Hablar de las cosas a través de la pintura}

No ha sido fácil llegar a la definición de pedagogía de la seducción como una capacidad que el o la docente ha de desenvolver con la finalidad de enseñar. Crean que se ha obviado muchos aspectos, de los cuales se es conscientes. Se sabe de la precipitación de llegar a esta definición, pero se considera oportuna para empezar a elaborar un mínimo de debate interno. Y, para ello, se propone explicar valiéndose de un recurso seductor: la pintura. Tal vez, se está poco acostumbrados a utilizar imágenes para darse a comprender o, incluso, para seducir. En ocasiones, son muy adecuadas; póngase el caso de los movies, las fotografías o la publicidad. No obstante, en esta ocasión, del medio que se irá a valer será la pintura de corte figurativa.

La estrategia didáctica se inspira no solo en hablar de pintura, sean de Goya o Rembrandt, sino en valerse de la pintura para empezar a seducir. Es decir, comprender una situación compleja en la cual se puede encontrar inmiscuidos pero que, al menos, se ha de atisbar alguna salida airosa. No vale con esconder la cabeza, en definitiva, la lucidez, entre el plumaje como haría un avestruz. La propuesta (a modo de fábula) se centra en comportarse como una jirafa que estira su largo cuello para otear el horizonte y prever la presencia de algún depredador, o bien conseguir alcanzar las ramas altas de aquel árbol para poder comérsela. Sin embargo, la altura de su cuello, parece que va en correspondencia con la longitud de sus piernas y, a veces, encuentra dificultad para beber agua en una superficie a ras de suelo. Pero ella es sabia y para solucionar la posible situación incómoda abre sus puertas y, curiosamente, deja de ver si algún depredador merodea su entorno y 
sus movimientos serán lentos hasta que no erija su pesado cuerpo. A pesar de todo, el riesgo lo debe correr. De lo contrario, la sed sería persistente.

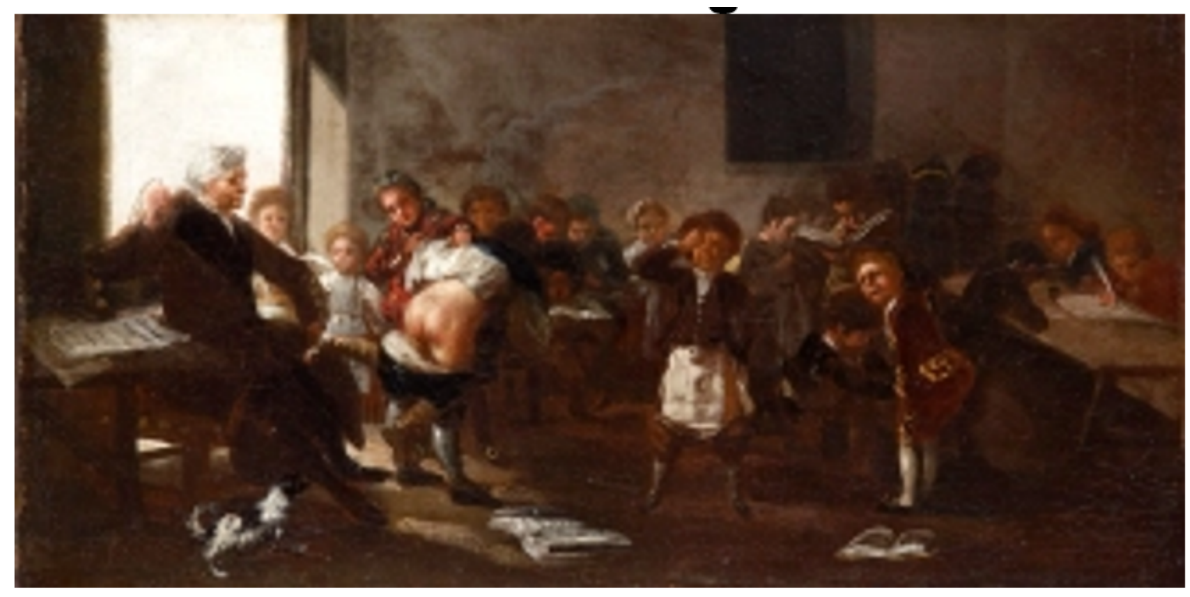

FIGURA 1

La letra con sangre entra

Fuente: Goya (1780-1785)

Pues bien, sin género de dudas, se cree que se ha consensuado que el fin de la letra con sangre entra y que se ha erradicado de todos los niveles educativos. A nadie se le pasa ni por la imaginación dar una azotina al alumnado que incomoda o se distrae en el aula, tal como se observa en la Figura 1. Goya lo pintó y e hizo pensar, tal vez, porque algunos hasta lo padecieron, lo que no se debe seguir permitiendo. Por la sencilla razón de que se trata de un delito, una atrocidad pedagógica y didáctica, además de que no garantiza nada, pues ¿quién experimenta en camisa ajena? Y el temor se apodera del aula, lejos queda la motivación, el interés... En definitiva, la seducción.

Es más, en cierto modo, se tendría que azotar en el trasero a todos, incluso a los que están atendiendo, pues en cualquier momento se pueden despistar y si no lo están, no crean que será por lo interesante del discurso del profesorado, quizás sea por la sospecha de que les puede tocar a alguno de ellos en cualquier descuido. No hay seducción y, por consiguiente, no existe el sinónimo de atracción. Lo sería más bien del miedo. El posible poder que pueda ejercer el o la docente se ha de traducir en liderazgo y fascinación, mientras que el pavor en el y la estudiante cabría descartarlo y se ha de diluir. No vale con mirar la acción aterradora del profesorado en la pintura de Goya ni, tampoco, es desinhibirse metiendo la cabeza entre las hojas del libro de clase, pues ahora a mí no me ha tocado. Es plantearse la acción pedagógica como algo entre las partes implicadas. En definitiva, es seducir: cautivando y encantando.

Tampoco el o la docente en esta pintura de Albert Anker seduce, tal como se puede observar en la Figura 2. No debe ser nada atractivo atender a un señor que en su mano derecha tiene una vara. Le falta la mano izquierda y, por ello, se vale del palo. Ciertamente, el alumnado le atiende. Se ve que son chicas y chicos y que al final del aula, la falta de atención cobra especial protagonismo. El profesorado cansado de tal actitud, decide centrarse en los que le prestan atención. Los escolares del fondo, en masa, dan la impresión de estar olvidados. El castigo, quizás, se traduzca en no prestarle demasiada atención y será un buen pretexto para, perpetuar, la desmotivación. Un desinterés que no solo se puede proyectar sobre el alumnado, también el profesorado tendrá su parcela de responsabilidad. 


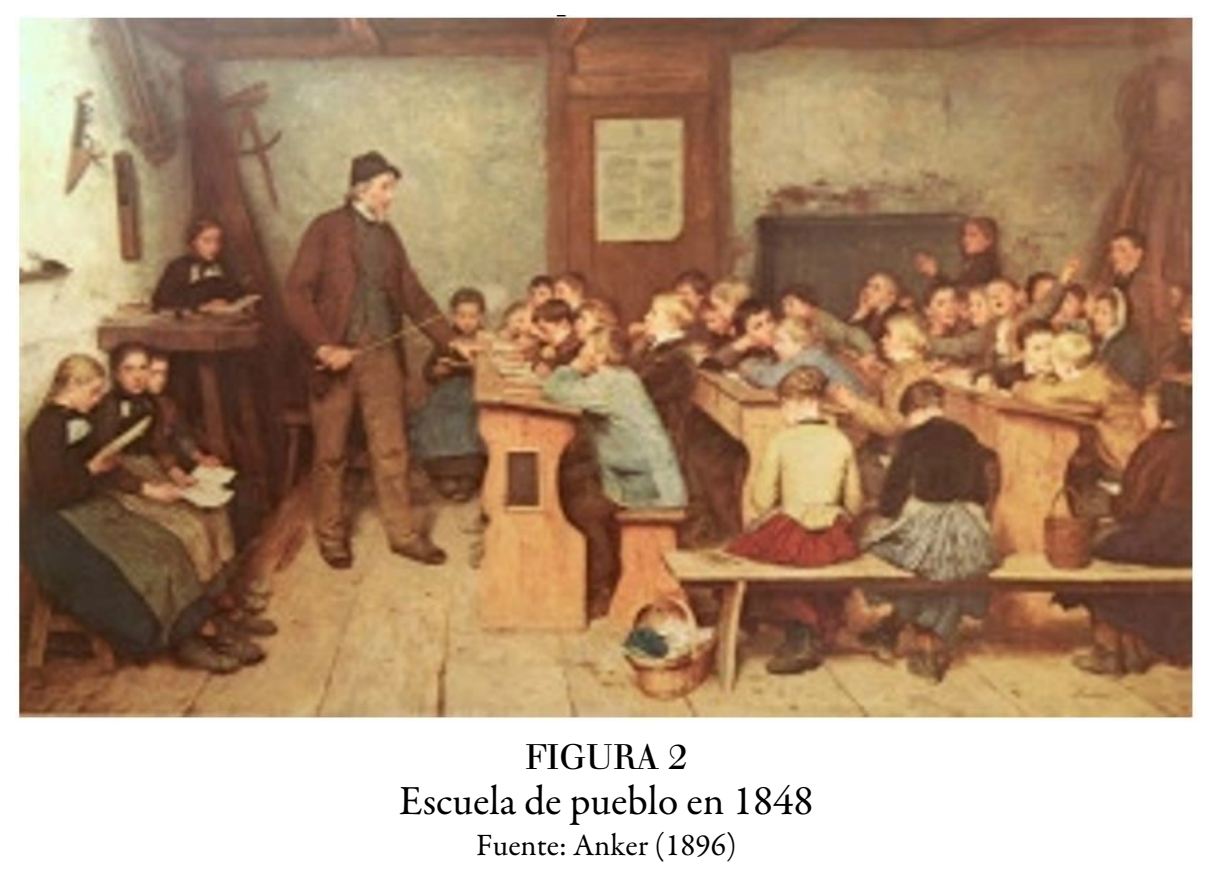

En este caso, sería muy fácil hablar de fracaso escolar, pero ¿cabría cuestionarse el fracaso profesional? Ahora bien ¿qué no se ha hecho para llegar a esta situación? Y lo más importante, ¿habría capacidad para remediarlo y no permitir que el grupo de alumnado que no atiende sea casi igual que los que en apariencia presta atención a las enseñanzas del o la docente?

Se está hablado del fin del o la docente, en exclusividad, como único referente. Costaría admitirlo, pero esta realidad se ha hecho evidente. Ahora se deben desarrollar nuevas responsabilidades en el aula. En este sentido, se estaría hablando de corresponsabilidades. E, incluso, se ha de propiciar estrategias para flexibilizar el agotamiento que, per se, promueve el aula, como lugar antinatura donde se aglutina a un sinnúmero de estudiantes y con las mejores de las intenciones se les enseña, a veces, si atender a la diversidad, sea de comportamientos, de intereses, o bien de ritmos de aprendizaje. Por ello, se propone pensar en el aula invertida (en inglés, flipped classroom) (Medina, 2016; Herrada y Baños, 2018). Es decir, donde antes se utilizaban el tiempo y el espacio del aula para explicar, ahora se usarían para trabajar en grupo y cuando se mandan tareas para casa, a partir de este momento, sería posible que el alumnado sea capaz de inquirir. $\mathrm{O}$ sea, aprender haciéndose preguntas.

El flipped learning trata en sí, de cómo hacer y la forma de interactuar con los alumnos, como permitir que estos desarrollen su potencial, como conseguir mantener la atención y la motivación extrínseca, ¿qué se puede hacer para mejorar sus competencias de la forma más autónoma?, ¿es posible presentar contenidos complejos de otro modo que no sea presencialmente?, todas estas preguntas e interrogantes y más, son las que en los últimos años se han estado haciendo docentes de todo el mundo y por ha existido una explosión el tema de metodología con una gran variedad de propuestas sobre metodologías activas de aprendizaje, cuyo uso llega desde educación infantil hasta la etapa Universitaria (Romero et al., 2019, p. 15). 


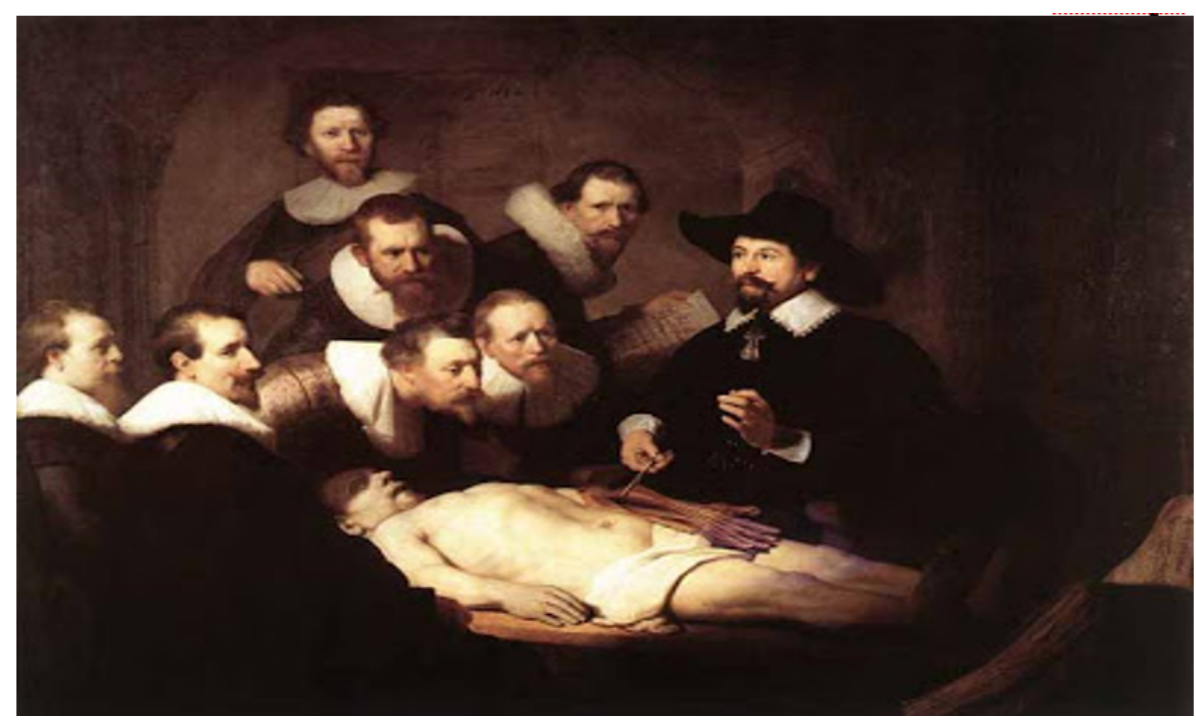

FIGURA 3

Clase de anatomía del Dr. Nicolás Tulp

Fuente: Rembrandt (1632)

Se presenta otro modelo de aula. Y, para ello, se vale de la pintura Clase de anatomía del Dr. Nicolás Tulp de Rembrandt, como pueden observar en la Figura 3. En este cuadro un médico expone su lección magistral ante un grupo de estudiantes, en este caso ya son adultos. El doctor ha de compartir el protagonismo de su voz con la mirada centrada sobre un cuerpo muerto, que es el motivo de atención de su explicación. El interés del alumnado está, completamente, dirigido; que se encuentra reforzado por el uso de la varita que porta en su mano izquierda que apunta hacia el brazo abierto y motivo de estudio. Mientras que el gesto de la mano derecha refuerza lo expuesto. Por parte del alumnado, no vale el menor de los despistes pues la información transmitida por la palabra se continúa con el proceder práctico de la clase. Aquí el o la docente tiene la responsabilidad de transmitir los conocimientos y el discente la de acompañarlos.

Se cuenta con cierto alumnado, en apariencia, pasivo que aprende a partir de la iniciativa del o la docente. En gran medida, la motivación se ha apoderado del alumnado que presta la mayor de las atenciones a la explicación práctica del o la docente. Unas y unos estudiantes de medicina que de forma autónoma acompañan la clase mientras uno de ellos, al final, constata la explicación del o la docente con unos apuntes. 


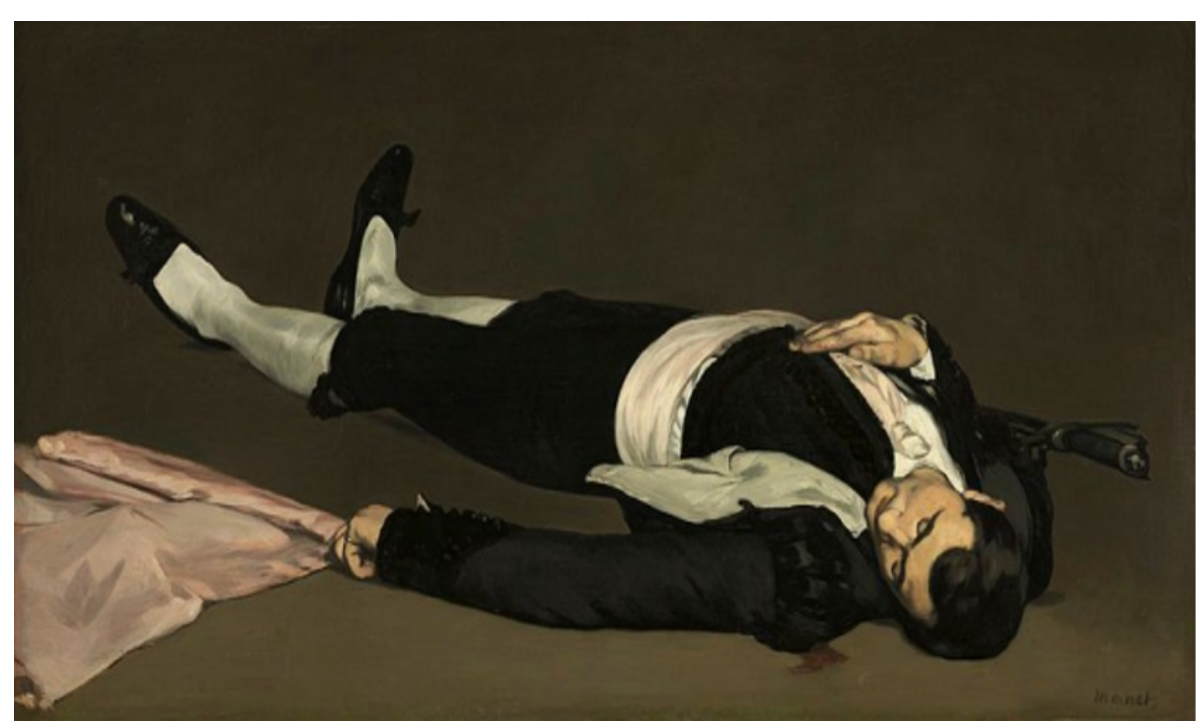

FIGURA 4

Torero muerto

Fuente: Manet (1864)

Para concluir sobre este epígrafe en relación con la enseñanza, ofreciéndole un protagonismo al personal docente, se irá a centrar sobre la majestuosa obra pictórica de la autoría de Edouard Manet, titulada Torero muerto (ver Figura 4). No solo muestra a un hombre que murió con sus enseres de matador de toros. Contemplando a este cuadro, se cuestiona si dejó escuela. Pues una vez el profesorado, nombre que también reciben los toreros experimentados, acaba su ciclo y su mérito se centra en los discípulos que le seguirán. De esta forma, sus enseñanzas se perpetúan en el tiempo, ya que el alumnado le recuerda. Y la satisfacción se colma cuando alguno de esos aprendices pueda llegar a superar al profesorado.

En cuatro pinturas se ha empezado a hablar de las cosas. Al fin y al cabo, ha sido un pretexto para continuar reflexionando sobre la figura del o la docente: sobre el castigo, en relación con la desmotivación, a propósito de la atención y, por último, quién seguirá al profesorado. En este caso, ha sido la manera de proseguir el debate de ideas sobre la necesidad de conocer, más y mejor, la pedagogía de la seducción.

\section{Pedagogía de la Seducción}

En la Universidad se están formando a personas para desenvolverse personal y profesionalmente para el futuro (García, 2009). El alumnado se hace adulto y se le responsabiliza, en parte, de su proceso formativo. La madurez y la autogestión casi se le exigen y es una atribución casi incuestionable. Ciertamente, el profesorado debe tener en cuenta que está en una etapa en que el y la estudiante es mayor de edad. No se les puede tratar como menores y, a veces, en lo relacionado con la formación no han conseguido aquel deseado nivel de autonomía y, en ocasiones, de capacidad de decisión. Por ello, el y la estudiante recurre al docente para que le vaya acompañando en su proceso, tal vez, añorando etapas educativas del pasado; utilizando los seminarios, las actividades en pequeño grupo o las tutorías. Estas últimas deberían ser:

entendida como como una parte de la responsabilidad docente en la que se establece una interacción personalizada entre el profesor y el estudiante con el objetivo de guiar el aprendizaje de este, adaptándolo a sus condiciones individuales y a su estilo de aprender, de modo que cada estudiante alcance el mayor nivel de dominio y competencia educativa posible. (García, 2008, p. 23).

La Universidad actual, a la cual se está haciendo mención, posee un marcado cariz práctico y, en el caso de las carreras sociales, por ejemplo, las vinculadas a la educación, habría que añadirle un sesgo profesional. 
Atrás quedó el o la docente con un profundo conocimiento a modo de saber atesorado, que se traducía en un educador bancario (Freire, 2005). En la actualidad, ha de adquirir la disposición de docente guía y dialogante, crítico y facilitador.

Ante un alumnado heredero de sus exigencias formativas en ciclos pasados y una institución universitaria para los nuevos tiempos, se habla de la pedagogía de la seducción como un quehacer que lo que pretende es plantear recursos para que el profesorado sepa acercarse y comprender a las personas y los contextos emergidos. Por ello, desde el momento en que se entra en el aula el o la docente debe tener en cuenta que su forma de proceder irá a interferir en la propia dinámica de aula. Todo lo relacionado con la comunicación no verbal (Hernández, 2013; Amar, 2014) serán recursos que le puede ayudar, y se diría que bastante. En este sentido, se habla de la próxemica. Es decir, el uso que el profesor/a universitario ha de hacer de las zonas; de los espacios existentes en el aula y que son disponibles para la enseñanza. Un ejercicio dinámico en el que se destaca desde la pertinencia a dejar en momentos puntuales su mesa a lo aconsejable de saber moverse entre los lugares en que se encuentra sentado el alumnado. A la vez, de la necesidad de establecer una buena capacidad de interconexión y de asentimiento con la mirada.

Prestando un mínimo de atención a sus estudiantes tendrá y obtendrá un excelente retorno de las sensaciones que estos puede ir teniendo de su clase. Ya que, observando sus rostros y su comunicación corporal conseguirá advertir si se está produciendo un acertado, o no, proceso de enseñanza-aprendizaje, si se encuentran motivados o, simplemente, les resulta interesante y no se aburren.

Por ello, se reivindica un docente cualificado. No vale tan solo con el que es capaz de transmitir narrativas interminables, sin dudas, bien hilvanadas. Ahora, para poner en acción la pedagogía de la seducción, hay que saber presentarla y conocer los principios que rigen la comunicación no verbal pues no solo tendrá que saber explicar sino, también, compartir protagonismo con las pantallas (Maroto, 2007; Mercader y Gairín, 2017). No solo con aquella que se proyecta para el grupo clase, sino, igualmente, sabiendo de la existencia de las muchas que el alumnado dispone para su uso personal. Al parecer, un docente dinámico entre los espacios del alumnado puede paliar el sobreuso de estos dispositivos inalámbricos, incluyéndolos en la dinámica de aula. A la vez que da la sensación de estar ante un profesorado más accesible que deja su mesa de docente para interactuar con los discentes y sus pertenencias inalámbricas, con la posibilidad de incluirla en la dinámica de clase.

Otra estrategia que se propone para este ejercicio por valorar la pedagogía de la seducción es todo lo relacionado con la manera de escuchar. Claro que se refiere, no solo la escucha de la palabra transmitida, que se ha de desarrollar de manera atenta. Si no, igualmente, de la escucha-comprensión de lo que sucede en el seno del aula. Para ello, se propone una especial consideración hacia la comunicación asertiva (Quiñonez y Moyano, 2019). Donde el principio de la comunicación como algo inherente al ser humano se nutre con el de establecer pensamientos, ideas o conocimientos de forma honesta, directa y apropiada. Facilitando la interacción, promoviendo el diálogo y manteniendo una escucha activa para perseguir la comprensión de lo que se enuncia y lo que sucede en el seno del aula. Una comunicación asertiva inspirada en la empatía, en la serenidad y en la escucha activa y, a la vez, respetuosa.

Este hecho comunicativo ha de evitar en el alumnado universitario cualquier tipo de ansiedad o malestar que pueda generar rechazo o desconexión con el relato del o la docente. En relación con la comunicación asertiva se proponen maneras proactivas que se inspiren en una mirada limpia, una actitud dialogante y un acercamiento al alumnado que pregunta u se observa que se encuentra con algún tipo de dificultad. Claro está que esta aproximación que se plantea no solo ha de ser física, puede obtenerse a través de la mirada, los gestos o el tono de voz. La intención sería la de salvar una situación que pueda perjudicar al estudiante en cuestión o al grupo clase. Con todo, la comunicación asertiva lo que pretende es establecer las bases para hacer del aula un espacio donde se faciliten las relaciones interpersonales, se incentive el consenso y se promueva un modelo de comunicación fluida y clara, adecuada y educada. 
Como último bastión sobre los que se asienta esto de la pedagogía de la seducción se centra en la capacidad que ha de desenvolver el o la docente universitario para admitir que ha de compartir cierto liderazgo con las pantallas (Abiétar, Belmonte y Giménez, 2018). Por ello, se propone pararse a pensar sobre su uso idóneo y que adquiera ciertas dotes coreográficas en el aula; en el sentido de arte y técnica que ayude a componer conjuntos pausados de movimientos del o la docente, con el propósito de centrar la atención en el alumnado. Produciendo en las y los estudiantes aquella añorada sensación de estar ante un profesorado capaz de adaptarse a los muchos papeles que le exige el guion de la profesión docente. Por ejemplo, con una mano señala lo más relevante de la pantalla mientras que, seguidamente, se dirige caminando entre el alumnado, de cara a ellos, para doblar el cuerpo y hablar mirando a la pantalla, cuando quiere destacar aquella idea importante. En el siglo XXI, y tras el advenimiento y desarrollo de las pantallas, se hace imprescindible saber utilizarlas (López, 2011) y admitir que han llegado para quedarse, también, entre el alumnado.

Es decir, se ha centrado el principio de la pedagogía de la seducción sobre tres bases: la comunicación no verbal, la comunicación asertiva y la coreografía entre pantallas. No obstante, se ha de reivindicar un arte cautivador, capaz de acercarse al otro. Es una manera de cautivar (en la acepción de prendido) antes de dejarlo cautivo (en el sentido de sometido). Es decir, un alumnado universitario que haciendo gala de la supuesta libertad que ha de tener y a la que ha de saber gestionar, venga a clase no por la obligación exclusiva que le exige la presencialidad sino, asimismo, por el interés por aprender en el aula y compartiendo. Unos y unas estudiantes que se sientan interesados por el conocimiento antes que obligado por el procedimiento universitario.

Pero, igualmente, la pedagogía de la seducción pasa por incentivar una mirada pedagógica anclada en el afecto y, a partir de esta estrategia didáctica, pretender conseguir la efectividad añorada de un aprendizaje eficiente. Es decir, tras el hecho cautivador y afectividad-efectividad, la pedagogía de la seducción promueve un arte por comprender al alumnado, del mismo modo que, establece las pautas para dar a comprender por parte del profesorado. Un juego sutil de miradas, gestos y propuestas narrativas que han de beneficiar los resultados de aprendizaje.

\section{No OLVIDAR QUE...}

Ciertamente, de un tiempo hacia acá, se ha producido una importante (re)evolución en todo lo relacionado con las tecnologías en todos los ámbitos de las vidas. Es decir, no se le escapa ninguna faceta, desde profesionales al entretenimiento (Rodríguez, Nieto y Sumozas, 2016). Y sobre este particular no se ha de olvidar o perder de vista de las futuras actuaciones, lo que se ha denominado como pedagogía de la seducción (Hermida, 2010; Morgado, 2011). En este sentido, no se puede ir contra la obviedad. Es innegable la profunda transformación que se ha experimentado en todo lo relacionado con la Universidad y que debería ser proporcional a la capacidad de cambio y de saber estar en conexión con los tiempos. Unas novedades que han venido para quedarse y que han de modificar la disposición con los actuales e innovadores momentos que ha tocado vivir y protagonizar en el siglo XXI.

Por ejemplo, en lo concerniente a la educación cabría señalar la emergencia del saber social que lleva a admitir la existencia de una ubicuidad que ha modificado el hecho de ponernos, simplemente, delante del conocimiento (Sevillano, González, Vázquez y Rey, 2016; Báez y Clunie, 2019). En cualquier lugar y en cualquier momento hace que el alumnado adquiera la posibilidad de aprender. Un profesorado universitario que ha de estar preparado en todo lo concerniente a los contenidos, pero igualmente, a las maneras de enseñarlos a través de la red. Ya no vale con la obsoleta y única lección magistral. Ya no debería ser necesaria u obligatoria la presencialidad. Todo se ha modificado y la creación de materiales virtuales ha de ser un motivo para aprender a expresarse con las herramientas tecnológicas (De Casas y Paramio, 2019).

Todo pasa por una (pre)disposición por admitir el cambio y activar todo lo más posible el quehacer de un docente innovador y con imaginación. Innovar como sinónimo de mejorar, de servicio a la comunidad y de 
cambio. Y una imaginación que se hace coincidir con tres verbos muy vinculados con la educación: a) crear, para promover aquello que no existe; b) mejorar, para optimizar en pro del beneficio y c) progresar, para experimentar un avance idóneo. Sin olvidarse de la necesidad de inquirir; de otorgarle al docente habilidades para que sea capaz de transmitir la necesidad de que el alumnado debe aprender preguntándose. No vale con la quietud que puede producirse, no en todos los casos, en el aula convencional. Se atisba unas pantallas que han de funcionar como aliadas, aunque las contemplemos, en algunos casos, como enemigas.

Por ello, se promueve un epígrafe donde no se olvide que todo está en continua construcción. Ante un panorama en y de cambio, el profesorado en este ejercicio de seducción en los entornos universitarios ha de ser dúctil y saber facilitar los ingredientes para que el alumnado pueda olvidarse de las recetas que le llevará a unos resultados previsibles. Se propone un docente universitario con predisposición a continuar aprendiendo, que sepa promover situaciones de aprendizaje y respete los diferentes ritmos de aprendizaje del discente (Zabalza, 2004; Monge y Gómez, 2018).

Siempre se suscribirá la necesidad de hacer de la pedagogía un arte y de la seducción una manera de aproximar. Con los pies en la tierra, sabiendo hacia dónde se va y hacia dónde se puede ir, se estará pendiente de los acontecimientos sociales, tecnológicos y de la educación superior (Varela et al., 2018). Con todo, se ha de poner las bazas para reconducir la mirada seductora sobre los contenidos y la manera de que estos se den a comprender.

\section{UN POSIBLE FINAL}

No es una conclusión, tampoco se trata de un resumen. La propuesta en este último epígrafe está centrada en seguir cuestionando el papel del o la docente universitario en esto que se ha venido llamando como la pedagogía de la seducción. No se vaya a permitir que prevalezcan inteligencias fracasadas (Marina, 2004). Probablemente, puedan que existan también pedagogías que promuevan el fracaso. Y, en este sentido, algo se tendrá que evitar. El o la docente universitario no puede asumir todas las responsabilidades sociales que llegan al aula. Ahora bien, ha de ser conscientes que existen cambios sociales y que su capacidad de transformación ha de ser importante para no defraudar. Mientras que el alumnado universitario maduro reivindica cualificación por parte del o la docente. $Y$, en este sentido, el profesor/a no puede defraudar. 


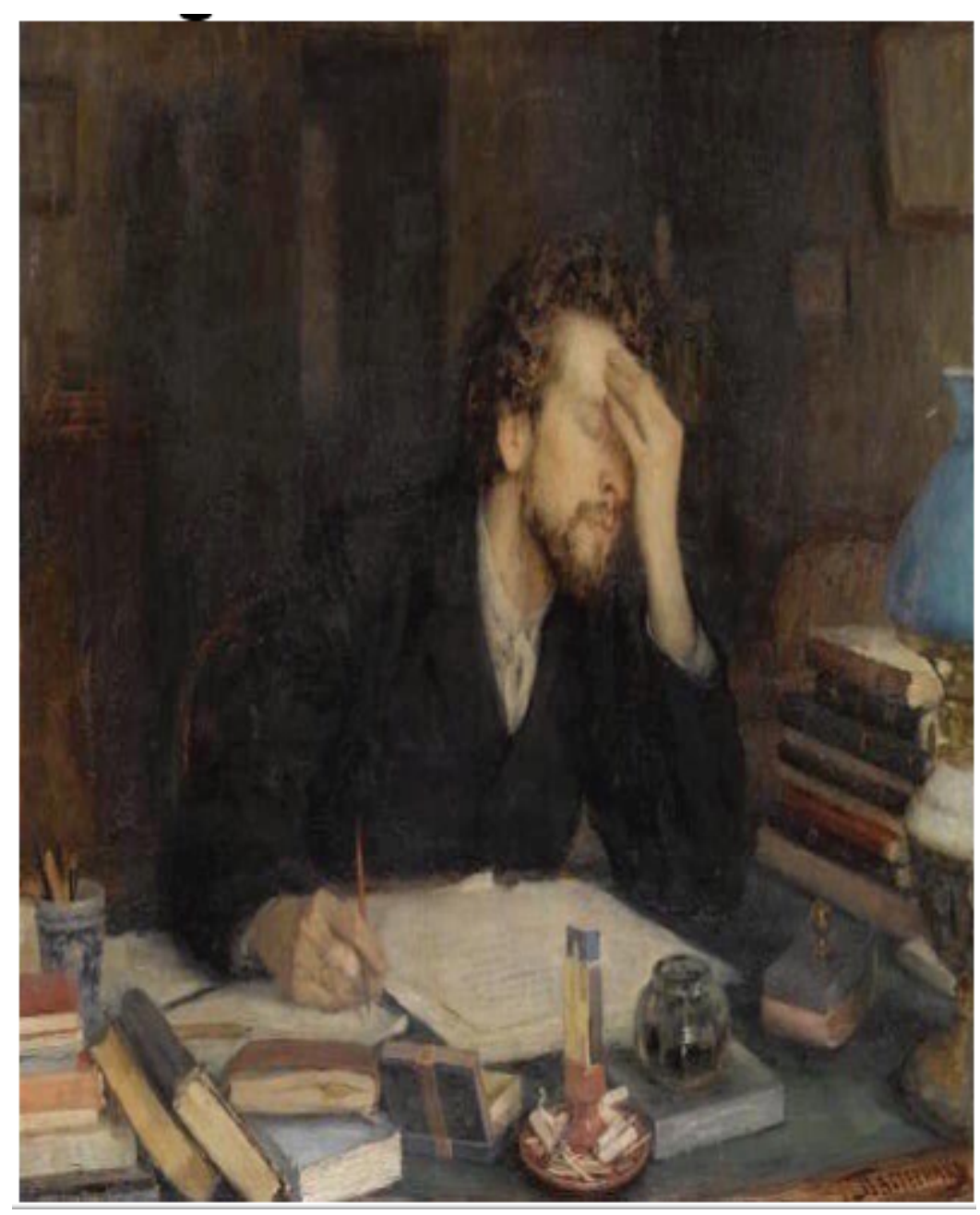

FIGURA 5

Agonía de la creación

Fuente: Pasternak (1899)

Sin abandonar la propuesta de recurrir a la pintura como vehículo de expresión y como hecho para dar a comprender, en este ejercicio compartido donde incluir la pedagogía de la seducción, se vale de dos cuadros que pueden invitar a continuar con el debate. Debe empezarse con la pintura de Leonid Pasternak, titulada Agonía de la creación (ver Figura 5). Un hombre frente a la responsabilidad de crear, pensativo y rodeado de libros.

La propuesta de pedagogía de la seducción no puede convertirse en una obsesión que precipite hacia la desesperación. Hay que ir, paulatinamente, madurándola. Es un proceso largo y continuado que tendrá sus frutos. En este sentido, los papeles han de estar sobre la mesa, junto con la predisposición al cambio (Del Valle y Calvo, 2011). Pero, en ningún momento, se ha de convertir en un tormento. La capacidad de adaptación ante los diferentes contextos, en cierto modo, será un buen indicador hacia este supuesto cambio. Y, se insiste en ello, la preparación del o la docente será otro aliciente más. Un trabajo repleto de conocimiento y adaptación, de lo contrario lo que en principio podría ser un ejercicio de innovación puede terminar convirtiéndose en una pesadilla, que no deja disfrutar del proceso y, en ocasiones, puede llegar a bloquear como docentes universitarios. 


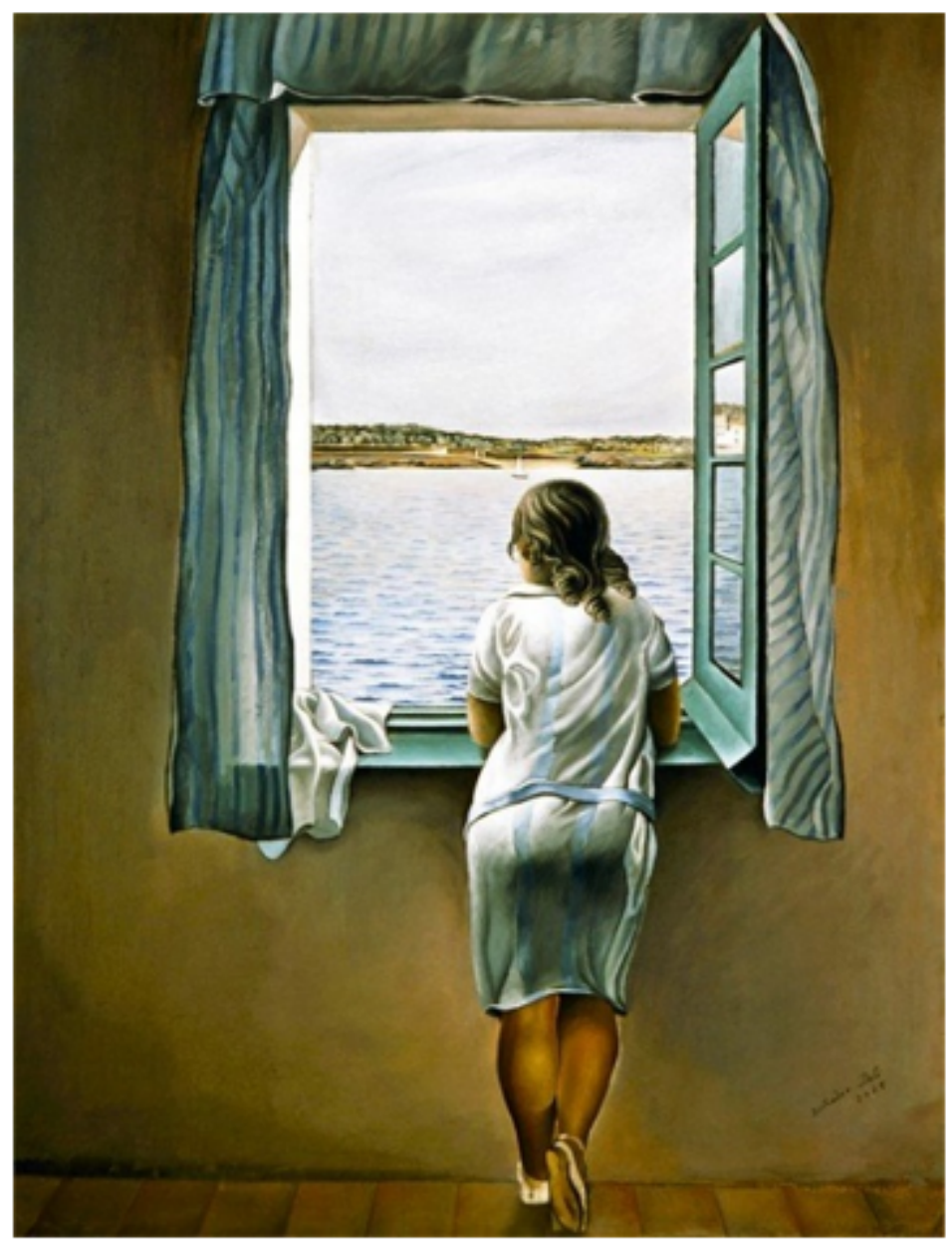

FIGURA 6

Muchacha en la ventana

Fuente: Dalí (1925)

En el otro lado de este ejercicio compartido estaría el alumnado. Este no se debe quedar mirando y esperando. En este caso, la inspiración pictórica para argumentarlo se centrará en el cuadro Muchacha en la ventana de Salvador Dalí (observar la Figura 6). La chica serena mira el panorama. Contempla y admira. Pero no vale con tan solo mirar, debe empezar a actuar. También, existe un importante elemento de implicación en este proceso que se ha compartido. Tal vez, le haga falta diferenciar entre admirar al exterior y mirar hacia el interior del aula. Es decir, sentirse protagonista. Y sin una actitud de colaboración sería difícil que se produzca la ansiada transformación. Es cierto que la iniciativa la ha de tomar cada docente; pero ha de estar en consonancia con la actitud de un alumnado, igualmente, predispuesto al cambio. Pues se ha de reivindicar que, todas y todos, son necesarios en esto de la pedagogía de la seducción.

\section{REFERENCIAS}

Abiétar, M, Belmonte, J. y Giménez, E. (2018). Educación, cultura y sociedad: Espacios críticos. Valencia: Tirant lo Blanch.

Alonso, S. y Alonso, M. (2014). Las redes sociales en las universidades españolas. Revista de la SEECI, 33, 132-140. doi: https://doi.org/10.15198/seeci.2014.33.132-140

Amar, V. (2014). Didáctica y comunicación no verbal. Salamanca: Comunicación Social. 
Anker, A. (1896). Escuela de pueblo en 1848 [Pintura]. Basilea. Basel Kunstmuseum. Recuperado de https://bit.ly/2 qspPA8

Báez, C. y Clunie C. (2019). Una mirada a la Educación Ubicua. RIED: revista iberoamericana de educación a distancia, 22(1), 325-344. doi: http://dx.doi.org/10.5944/ried.22.1.22422

Brazuelo, F. (2015). Recursos móviles y realidad aumentada. En M. Cacheiro, C. Sánchez y J. González (Coord.). Recursos tecnológicos en contextos educativos (pp. 399-432). Madrid: UNED.

Brazuelo, F., Gallego, D. y Cacheiro, M. (2017). Los docentes ante la integración educativa del teléfono móvil en el aula. RED. Revista de Educación a Distancia, 52, 1-22. Recuperado de http://www.um.es/ead/red/52

Carrasco, J. (2015). Motivar para educar. Madrid: Narcea.

Ceprián, J. (2019). Tecnología y educación, compañeras de viaje. Cuadernos de pedagogía, 500, 123-127.

Dalí, S. (1925). Muchacha en la ventana [Pintura]. Madrid. Museo Reina Sofía. Recuperado de https://bit.ly/2Rm $\mathrm{NcpK}$

De Casas, P. y Paramio, G. (2019). Educación y comunicación mediada por las tecnologias: tendencias y retos de investigación. Sevilla: Egregius.

Del Valle, M. y Calvo, E. (2011). Cambios en los modelos educativos en el EEES. Madrid: Visión Libros.

Díaz, M. (2019). ¿Qué es eso que se llama pedagogía? Pedagogía y saberes, 50, 11-28.

Ertmer, P. y Ottenbreit, A. (2010). Teacher Technology Change: How Knowledge, Confidence, Beliefs, and Culture Intersect. Journal of Research on Technology in Education, 42(3), 255-284. Recuperado de https://files.eric.ed.g ov/fulltext/EJ882506.pdf

Freire, P. (2005). Pedagogía del oprimido. México: Siglo XXI.

García, J. (2009). Futuro de la Universidad o Universidad del futuro. Fuentes: Revista de la Facultad de Ciencias de la Educación, 9, 9-25.

García, N. (2008). La función tutorial de la Universidad en el actual contexto de la Educación Superior. Revista Interuniversitaria del Profesorado, 22(1), 21-48.

Goya, F. (1780-1785). La letra con sangre entra [Pintura]. Zaragoza. Museo de Zaragoza. Recuperado de https://bit .ly/2OQISNK

Grene, R. (2008): El arte de la seducción. Madrid: Espasa.

Hermida, J. (2010). Hablar sin palabras: cómo dominar todas las situaciones a través de los gestos. Barcelona: Temas de hoy.

Hernández, M. (2013). Comunicación no verbal y educación: el comportamiento kinésico en el ámbito universitario. En J. Esteban (Ed.). Marcas del cuerpo en educación: imaginarios simbólicos y materiales (pp. 77-88). Valladolid. Universidad Europea Miguel de Cervantes.

Herrada, R. y Baños, R. (2018). Revisión de la clase invertida en la docencia universitaria. En A. Rosa-García (Coord.), Aplicando la clase invertida en empresa, economia y turismo (pp. 27-36). Madrid: Dykinson.

Krücken, G. (2014). Higher Education Reforms and Unintended Consequences: A Research Agenda. Studies in Higher Education, 39(8), 1439-1450. doi: https://doi.org/10.1080/03075079.2014.949539

Lazos, M. y Gabelas, J. (2016). Comunicación digital: un modelo basado en el Factor R-Elacional. Barcelona: UOC.

López, M. (2011). Educación para el siglo XXI. Bilbao: Desclée De Brouwer.

Manet, E. (1864). Torero muerto [Pintura]. Washington DC. National Gallery of Art. Recuperado de https://histor ia-arte.com/obras/manet-torero-muerto

Marcelo, C., Yot, C. y Mayor, C. (2015). Enseñar con tecnologías digitales en la Universidad. Comunicar, 45, 117-124. doi: https://doi.org/10.3916/C45-2015-12

Marina, J. (2004). La inteligencia fracasada. Barcelona: Anagrama.

Maroto, A. (2007). El uso de las nuevas tecnologías en el profesorado universitario. Pixel-bit. Revista de medios $y$ educación, 30, 61-72. Recuperado de https://idus.us.es/bitstream/handle/11441/45634/file_1.pdf?sequence= 18isAllowed=y 
Medina, J. (2016). La docencia universitaria mediante el enfoque del aula invertida. Barcelona: Octaedro.

Meirieu, P. (1987). Aprender, sí. Pero ¿cómo? Barcelona: Octaedro.

Meirieu, P. (2018). Pedagogía: necesidad de resistir. Madrid: Popular.

Mercader, C. y Gairín, J. (2017). ¿Cómo utiliza el profesorado universitario las tecnologías digitales en sus aulas? REDU: Revista de Docencia Universitaria, 15(2), 257-274. doi: https://doi.org/10.4995/redu.2017.7635

Monge, C. y Gómez, P. (2018). Innovando la docencia desde la formación del profesorado. Madrid: Síntesis.

Morgado, M. (2011). Autoridade e sedução na relação pedagógica. Revista Psicologia da educação, 32, 113-130. Recuperado de http://pepsic.bvsalud.org/scielo.php?script=sci_arttext\&pid=S1414-69752011000100007\&l $\mathrm{ng}=\mathrm{pt} \& \mathrm{t} \operatorname{lng}=\mathrm{pt}$.

Pasternak, L. (1899). Agonía de la creación [Pintura]. San Petersburgo. Museo Ruso. Recuperado de https://bit.ly/2 $\operatorname{Lrj} 7 \mathrm{Bx}$

Perrenoud, Ph. (2001). Dévolopper la practique réflexive dans le métier d'enseignant. Professionalisation et raison pédagogique. París: ESF editeur.

Quiñonez, J. y Moyano, G. (2019). La asertividad como estilo de comunicación en la formación del sujeto educador. Revista Scientific, 4, 68-83. doi: https://doi.org/10.29394/Scientific.issn.2542-2987.2019.4.E.4.68-83

Rembrandt, H. (1632). Clase de anatomia del Dr. Nicolás Tulp [Pintura]. La Haya. Museo Mauritshuis. Recuperado de https://bit.ly/2qmRDFW

Rodríguez, M., López, A. y Martín, I. (2017). Percepciones de los estudiantes de Ciencias de la Educación sobre las redes sociales como metodología didáctica. Pixel-Bit: Revista de medios y educación, 50, 77-93. doi: http://dx.d oi.org/10.12795/pixelbit.2016.i50.05

Rodríguez, M., Nieto, E. y Sumozas, R. (2016). Las tecnologias en educación. Madrid: Síntesis

Romero, V., Romero, M., Toala, F., Castro, J., Pin, Á., Campozano, Y. y Gruezo, O. (2019). El flipped learning, el aprendizaje colaborativo y las herramientas virtuales en la educación. Alcoy: 3 Ciencias.

Ruiz, D. (2006). La formación docente: entre la indiferencia y la seducción Educere, 10(32), 49-54. Recuperado de h ttp://www.redalyc.org/articulo.oa?id=35603208

Sevillano, M., González, M., Vázquez, E. y Rey, L. (2016). Ubicuidad y movilidad de herramientas virtuales abren nuevas expectativas formativas para el estudiantado universitario. Ensayos Pedagógicos, 11(2), 99-131. Recuperado de https://www.revistas.una.ac.cr/index.php/ensayospedagogicos/article/view/9149/10674

Zabalza, M. (2004). La enseñanza universitaria: El escenario y sus protagonistas. Madrid: Narcea.

CC BY-NC-ND 